\title{
Thermodynamic determination of fragility in La-based glass-forming liquid
}

\author{
JIA Ran, BIAN XiuFang ${ }^{*}$ \& WANG YingYing \\ Key Laboratory for Liquid-Solid Structural Evolution and Processing of Materials of Ministry of Education, Shandong University, Jinan 250061, \\ China
}

Received May 31, 2011; accepted September 23, 2011

\begin{abstract}
Differences in the thermodynamic functions between the liquid and crystalline states of La-based bulk metallic glasses alloys were calculated with the specific heat capacity $C_{\mathrm{p}}$ and the fusion heat $\Delta H_{\mathrm{f}}$, which we measured. Fragility indexes having different thermodynamic definitions were calculated from the temperature dependence of excess entropy $\Delta S^{\text {liq-cry }}$. It is ambiguous for La-based glass-forming liquid to evaluate fragility from the intercepts of $\Delta S^{\text {liq-cry }}$-temperature curves. We found that the thermodynamic fragility $M_{\Delta S}$, denoted $\Delta S$, decreases at the onset melting temperatures $T_{\mathrm{m}}$ rather than at glass transition temperatures $T_{\mathrm{g}}$, and relates linearly with the kinetic fragility $m$. The correlations between thermodynamic fragility and kinetic fragility are discussed within the frameworks of the Adam-Gibbs relationship and potential energy landscape theory.
\end{abstract}

thermodynamics, fragility, La-based bulk metallic glasses, heat capacity

Citation: Jia R, Bian X F, Wang Y Y. Thermodynamic determination of fragility in La-based glass-forming liquid. Chinese Sci Bull, 2011, 56: 3912-3918, doi: 10.1007/s11434-011-4844-x

The concept of liquid fragility has been widely used to classify the dynamic behavior of glass-forming liquids since first being introduced by Angell in 1985 [1]. According to the concept, glass-forming liquids are classified between two extremes: strong glass formers showing Arrhenius behavior and fragile glass formers that deviate from Arrhenius behavior. However, the fragility parameter $m$ can only be calculated for systems with stable undercooled liquid. Recently, a new concept, the fragility of superheated melts $M$, defined as the rate of variation of the viscosity of superheated liquids towards the liquidus temperature, was proposed by Bian et al. [2]. A negative correlation between $M$ and glass-forming ability (GFA) has been found in Al-based [2-4] and rare-earth-based alloy systems [5,6]. Moreover, the correlation between $m$ and $M$ has also been investigated $[3,7]$.

The two fundamental aspects of phase transition are thermodynamics and dynamics. The Kauzmann paradox

*Corresponding author (email: xfbian@sdu.edu.cn) suggests that the two are inherently related [8]. The Adam and Gibbs model [9], which is based on cooperatively relaxing regions in supercooled liquids, provided the first quantitative relation between the thermodynamic and kinetic properties of a liquid. Several attempts to correlate the fragility (the kinetic property) of supercooled liquids with thermodynamic behaviors have been made, and have mainly focused on changes in heat capacity [10] and entropy [11-14]. However, the thermodynamic fragility of metallic glasses has not been investigated systematically [11,14-17].

Heat capacity is the most fundamental thermodynamic nature of matter. In this paper, the specific heat capacity of six La-based bulk metallic glasses (BMGs) is measured for the crystalline solid, the supercooled liquid, and the superheated liquid. The differences in thermodynamic functions between the liquid and the crystalline states are calculated. With these data, the thermodynamic fragilities defined previously $[12,14]$ are calculated and new thermodynamic fragility indexes are proposed to investigate the interconnection of thermodynamics and kinetic fragility. 


\section{Experimental procedure}

Ingots with nominal compositions of $\mathrm{La}_{55} \mathrm{Al}_{25} \mathrm{Ni}_{20}$ (La-1), $\mathrm{La}_{55} \mathrm{Al}_{25} \mathrm{Ni}_{15} \mathrm{Cu}_{5}$ (La-2), $\mathrm{La}_{55} \mathrm{Al}_{25} \mathrm{Ni}_{5} \mathrm{Cu}_{15}$ (La-3), $\mathrm{La}_{60} \mathrm{Al}_{10^{-}}$ $\mathrm{Cu}_{20} \mathrm{Ni}_{10}$ (La-4), $\mathrm{La}_{55} \mathrm{Al}_{25} \mathrm{Cu}_{10} \mathrm{Co}_{5} \mathrm{Ni}_{5}$ (La-5), and $\mathrm{La}_{62} \mathrm{Al}_{14}$ $\mathrm{Cu}_{12} \mathrm{Ni}_{12}$ (La-6) were prepared by arc-melting with $99.9 \mathrm{wt} \%-$ $99.999 \mathrm{wt} \%$ pure $\mathrm{Al}, \mathrm{Co}, \mathrm{Cu}, \mathrm{Ni}$ and industrially pure $\mathrm{La}$ in a Ti-gettered argon atmosphere. To ensure the homogeneity of the samples, the ingots were remelted at least three times in addition to being electromagnetically whisked. BMG rods with a diameter of $2 \mathrm{~mm}$ and a length of $50 \mathrm{~mm}$ were prepared by copper-mould suction casting in an arc furnace. The amorphous structure (except in the case of La-1) of the transverse cross sections of the rods was identified by X-ray diffraction using a D/Max-rB diffractometer and $\mathrm{Cu} \mathrm{K \alpha}$ radiation.

A Netzsch DSC404 differential scanning calorimeter (DSC) was used to determine the characteristic temperature and the specific heat capacity of the alloys. For heat-flux differential scanning calorimetry, the resultant signal is expressed as

$$
Q=K \cdot \mathrm{DSC}=C_{\mathrm{p}} \cdot m \cdot H R,
$$

where $K$ is the sensitivity coefficient, DSC is the measurement result, $C_{\mathrm{p}}$ is the specific heat capacity of unit mass, $m$ is the sample mass and $H R$ is the heating rate. With the same heating rate $H R$, the measured absolute heat flow minus the baseline (the heat flow of the pan itself) is directly proportional to the heat capacity $\left(C_{\mathrm{p}} \cdot m\right)$, and it can be expressed at a certain temperature as

$$
\frac{Q_{\text {sam }}-Q_{\mathrm{pan}}}{Q_{\mathrm{std}}-Q_{\mathrm{pan}}}=\frac{C_{\mathrm{p}}(T)_{\mathrm{sam}} \cdot m_{\mathrm{sam}}}{C_{\mathrm{p}}(T)_{\mathrm{std}} \cdot m_{\mathrm{std}}},
$$

where $m$ is the mass in the unit of grams and $C_{\mathrm{p}}$ is the specific heat capacity in the unit of Joules per gram per degree Kelvin. The subscripts sam, std and pan indicate the metal sample, the sapphire standard and the sample pan respectively.

At a heating rate of $20 \mathrm{~K} / \mathrm{min}$, by successively performing calorimetric measurements on a sample pan by itself, on a sapphire standard in the sample pan and on the metal sample in the sample pan, the absolute specific heat capacity of the metal sample is determined according to

$$
C_{\mathrm{p}}(T)_{\mathrm{sam}}=\frac{\left(Q_{\mathrm{sam}}-Q_{\mathrm{pan}}\right) \cdot C_{\mathrm{p}}(T)_{\mathrm{std}} \cdot m_{\mathrm{std}}}{\left(Q_{\mathrm{std}}-Q_{\mathrm{pan}}\right) \cdot m_{\mathrm{sam}}} .
$$

The mass of each sample is approximately $30 \mathrm{mg}$. The amorphous rods were heated nearly to their melting temperatures $T_{\mathrm{m}}$, determined beforehand using the DSC, for $C_{\mathrm{p}}$ measurements and then cooled to room temperature freely to form the crystallized samples. The crystallized samples were heated to $\sim 200 \mathrm{~K}$ above starting melting temperatures to obtain the specific heat capacity of both the crystallized solid and liquid metals. During heating, all samples were first heated to $373 \mathrm{~K}$ and held isothermally for $10 \mathrm{~min}$ and then heated at a constant rate of $20 \mathrm{~K} / \mathrm{min}$ for $C_{\mathrm{p}}$ measurements. Each experiment was carried out under a high-purity argon atmosphere.

\section{Results}

\subsection{Thermodynamics}

Upon heating the amorphous alloys at a constant rate, the alloys go through glass transition, crystallization, and fusion. Such behavior of $\mathrm{La}_{55} \mathrm{Al}_{25} \mathrm{Ni}_{5} \mathrm{Cu}_{15}$ (La-3) is shown in Figure 1 as an example. By integrating the area of the melting event (from solidus temperature $T_{\mathrm{m}}$ to liquidus temperature $T_{1}$ ), the total heat of fusion $\Delta H_{\mathrm{f}}$ is calculated. The entropy of fusion can then be estimated as $\Delta S_{\mathrm{f}}=\Delta H_{\mathrm{f}} / T_{\mathrm{f}} . T_{\mathrm{f}}$ is the temperature at which the Gibbs free energy of the crystal is equal to that of the liquid. $T_{\mathrm{f}}$ between $T_{\mathrm{m}}$ and $T_{1}$ is likely to be very close to the melting start temperature $T_{\mathrm{m}}$. Thus, $T_{\mathrm{m}}$ is adopted as $T_{\mathrm{f}}$ in calculating $\Delta S_{\mathrm{f}}$. The characteristic temperatures and the heats and entropies of fusion of these Labased alloys are summarized in Table 1. $T_{\mathrm{g}}$ of La-1, for which we did not obtain a fully amorphous rod, is cited from [18]. There are slight differences in characteristic temperatures between our work and [18-21], as is usually the case for different raw materials and laboratory facilities.

The measured specific heat capacity is shown in Figure 2. The heat capacity of glass is similar to that of crystal at low temperatures. However, when heated above the glass transition temperature $T_{\mathrm{g}}$, the glass phase relaxes into the supercooled liquid state and its $C_{\mathrm{p}}$ value assumes the higher heat capacity of a liquid [22]. Inoue et al. [21] and $\mathrm{Lu}$ et al. [20] reported that the specific heat capacity of $\mathrm{La}_{55} \mathrm{Al}_{25} \mathrm{Ni}_{10} \mathrm{Cu}_{10}$ alloy is 42.0 and $39-41 \mathrm{~J} \mathrm{~mol}^{-1} \mathrm{~K}^{-1}$ respectively throughout the undercooled liquid region; our measurement results are similar. $C_{\mathrm{p}}$ values for several La-based BMGs have so far been reported [21]; however, this is the first time that $C_{\mathrm{p}}$ for

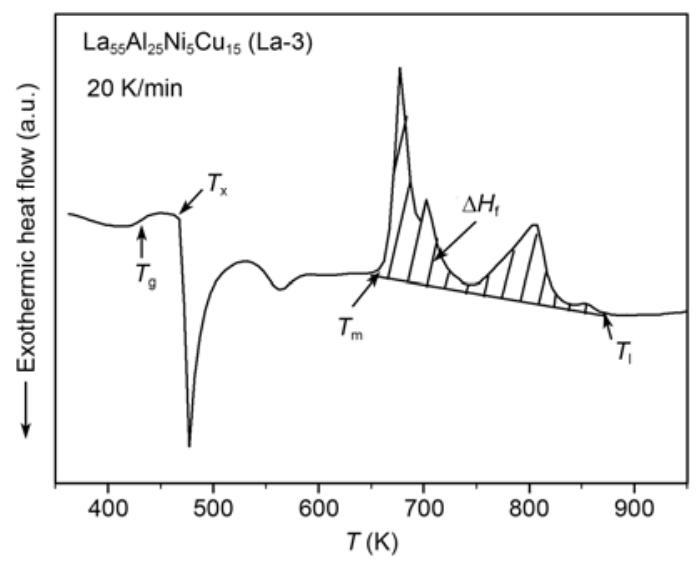

Figure 1 DSC heat flow curve of La-3 at a heating rate of $20 \mathrm{~K} / \mathrm{min}$. 
Table 1 Characteristic temperatures and the heats and entropies of fusion of La-based alloys

\begin{tabular}{clcccccc}
\hline Alloys & $T_{\mathrm{g}}(\mathrm{K})$ & $T_{\mathrm{m}}(\mathrm{K})$ & $T_{1}(\mathrm{~K})$ & $\Delta H_{\mathrm{f}}\left(\mathrm{kJ} \mathrm{mol}^{-1}\right)$ & $\Delta S_{\mathrm{f}}\left(\mathrm{J} \mathrm{mol}^{-1} \mathrm{~K}^{-1}\right)$ & $T_{\mathrm{k}}(\mathrm{K})$ \\
\hline La-1 & $491^{\mathrm{a}}$ & 700 & 950 & 7.612 & 10.9 & 339 \\
La-2 & 447 & 661 & 873 & 6.220 & 7.41 & 343 \\
La-3 & 433 & 656 & 828 & 773 & 7.948 & 10.7 & 320 \\
La-4 & 412 & 680 & 887 & 7.744 & 11.7 & 329 \\
La-5 & 492 & 665 & 758 & 8.084 & 12 & 356 \\
La-6 & 410 & 671 & & & & 292 \\
\hline
\end{tabular}

a) From Ref. [18].

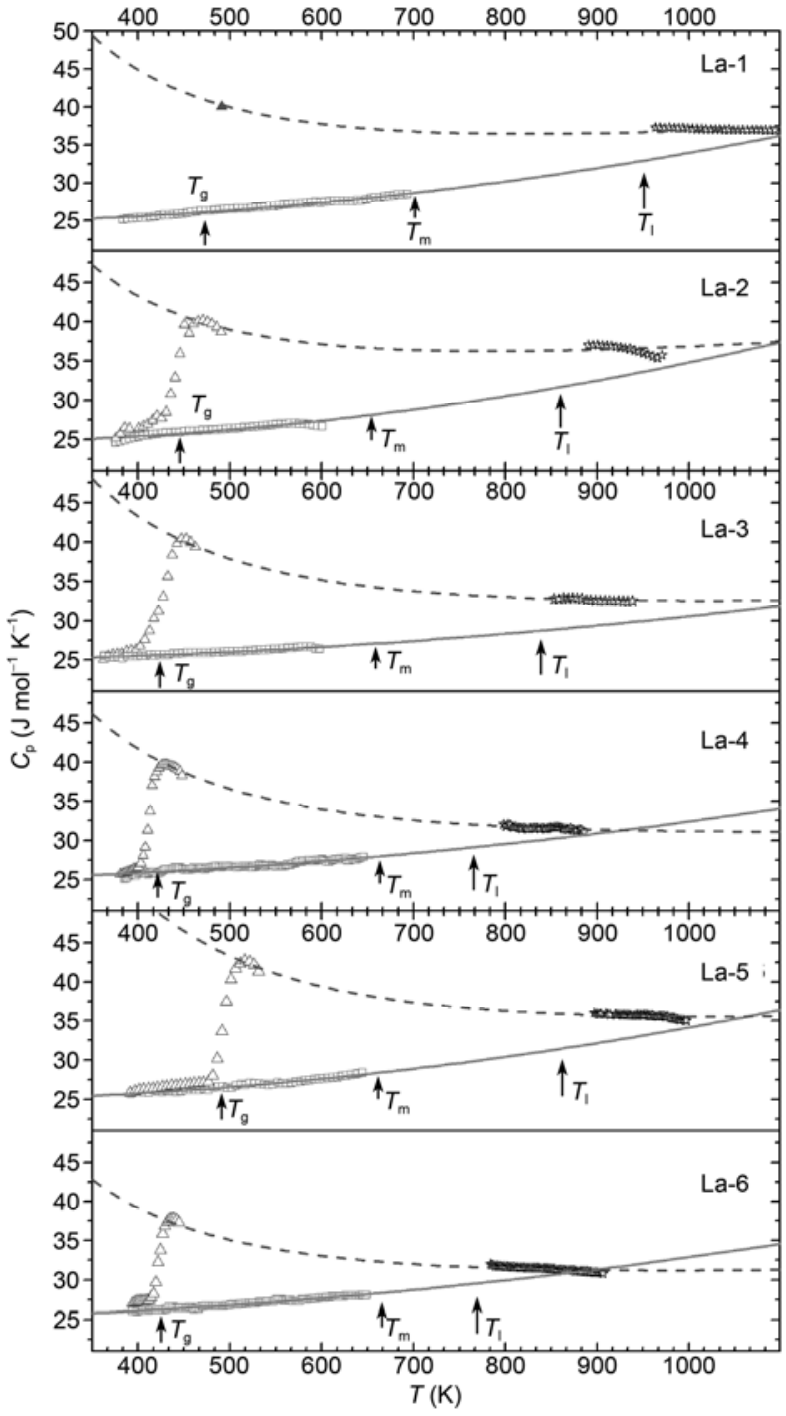

Figure 2 DSC heat capacity measurements obtained from initially liquid (次), crystalline ( $\square$ ) and glassy $(\triangle)$ samples, and the fitting lines of eq. (4) (solid lines) and eq. (5) (dashed lines). For La-1, the glassy data were not measured, but a value of $40 \mathrm{~J} \mathrm{~mol}^{-1} \mathrm{~K}^{-1}$ at $500 \mathrm{~K}, 10 \mathrm{~K}$ higher than $T_{\mathrm{g}}$, was assigned to allow the determination of the fitting constant for the liquid specific heat capacity $(\mathbf{\Lambda})$.

the both supercooled liquid and the melts has been measured to ensure fitting of the following eq. (5).

The heat capacity of a crystal well above the Debye temperature can be described by [23]

$$
C_{\mathrm{p}}^{\mathrm{cry}}(T)=3 R+a T+b T^{2}
$$

and the heat capacity of a liquid can be described by

$$
C_{\mathrm{p}}^{\mathrm{liq}}(T)=3 R+c T+d T^{-2},
$$

where the gas constant $R=8.3145 \mathrm{~J} \mathrm{~mol}^{-1} \mathrm{~K}^{-1}$, and $a, b, c$ and $d$ are fitting constants. The fitting curves and $T_{\mathrm{m}}$ and $T_{1}$ are shown in Figure 2. If an equilibrium liquid continuously cools to a supercooled liquid without solidification, which is required in glass formation, $C_{\mathrm{p}}$ does not abruptly change and the $C_{\mathrm{p}}$ curve throughout the equilibrium and supercooled range coincides with eq. (5) [21,24-27]. Table 2 summarizes the fitting constants for both eqs. (4) and (5).

The specific heat capacity of La-1 $\left(\mathrm{La}_{55} \mathrm{Al}_{25} \mathrm{Ni}_{20}\right)$ in the undercooled liquid that initially forms upon heating BMGs could not be measured since a fully amorphous rod was not obtained. Of the other five alloys in this work, the heat capacity is highest at a temperature about $10 \mathrm{~K}$ higher than $T_{\mathrm{g}}$, and it has an average value of about $40 \mathrm{~J} \mathrm{~mol}^{-1} \mathrm{~K}^{-1}$. The glass transition temperature $T_{\mathrm{g}}$ of $\mathrm{La}_{55} \mathrm{Al}_{25} \mathrm{Ni}_{20}$ is reported to be $490 \mathrm{~K}$ [18]. Thus, we assume a data point of $40 \mathrm{~J} \mathrm{~mol}^{-1} \mathrm{~K}^{-1}$ at $500 \mathrm{~K}$ for the data fitting of the liquid specific heat capacity.

Since the heat capacity of liquid and crystalline states and the enthalpy of fusion $\Delta H_{\mathrm{f}}$ have been experimentally determined, the enthalpy and entropy differences between the liquid and crystalline states of the alloy can be calculated as functions of temperature:

$$
\begin{gathered}
\Delta H^{\text {liq-cry }}(T)=\Delta H_{\mathrm{f}}-\int_{T}^{T_{\mathrm{f}}} \Delta C_{\mathrm{p}}^{\text {liq-cry }}\left(T^{\prime}\right) \mathrm{d} T^{\prime}, \\
\Delta S^{\text {liq-cry }}(T)=\Delta S_{\mathrm{f}}-\int_{T}^{T_{\mathrm{f}}} \frac{\Delta C_{\mathrm{p}}^{\text {liq-cry }}\left(T^{\prime}\right)}{T^{\prime}} \mathrm{d} T^{\prime},
\end{gathered}
$$

where $\Delta C_{\mathrm{p}}^{\text {liq-cry }}=C_{\mathrm{p}}^{\text {liq }}-C_{\mathrm{p}}^{\text {cry }}$. As mentioned above, $T_{\mathrm{f}}$ is substituted with $T_{\mathrm{m}}$ for the calculation. Figure 3 shows the calculated differences in the enthalpy and entropy between the liquid and crystalline states of La-1 as an example. On the plot, we mark $T_{\mathrm{g}}, T_{\mathrm{m}}$ and the calculated Kauzmann temperature $T_{\mathrm{k}}$. At $T_{\mathrm{k}}$, the entropy of the liquid is equal to the entropy of the crystal. The values of $T_{\mathrm{k}}$ are also listed in Table 1. 
Table 2 Fitting constants for the heat capacity data, according to eq. (4) for crystalline states and eq. (5) for liquid

\begin{tabular}{cccc}
\hline Alloys & $a\left(10^{-3} \mathrm{~J} \mathrm{~mol}^{-1} \mathrm{~K}^{-2}\right)$ & $b\left(10^{-5} \mathrm{~J} \mathrm{~mol}^{-1} \mathrm{~K}^{-2}\right)$ & $c\left(10^{-3} \mathrm{~J} \mathrm{~mol}^{-1} \mathrm{~K}^{-2}\right)$ \\
\hline La-1 & -3.30 & 1.23 & 9.34 \\
La-2 & -4.74 & 1.46 & 9.61 \\
La-3 & -1.36 & 0.697 & 5.00 \\
La-4 & -5.79 & 0.156 & 3.78 \\
La-5 & -2.68 & 1.19 & 6.87 \\
La-6 & -0.443 & 0.836 & 2.31 \\
\hline
\end{tabular}

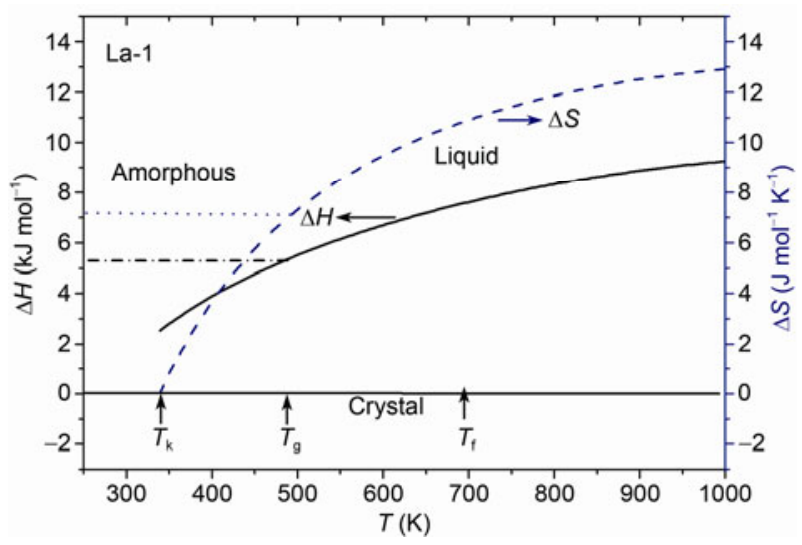

Figure 3 Calculated differences in enthalpy (solid lines) and entropy (dash lines) between the liquid and crystalline states of La-1.

\subsection{Fragility}

Besides consideration of the viscosity, thermal scanning is thought to be a reliable way to obtain the fragility of the supercooled liquid [28]. Previous studies have demonstrated that the kinetic heating rate $\varphi$ dependence of the glass transition temperature $T_{\mathrm{g}}$ describes the fragility as well as the temperature dependence of viscosity [29]. Values of $m$ for La-1, La-2 and La-3, determined using the method employed in [6], are cited from [18], while values for the other three alloys were obtained employing the same method used in the DSC experiments at different heating rates; values are presented in Table 3.

Ito et al. [12] used a Kauzmann plot to define the thermodynamic fragility of glass-forming liquids, and found that thermodynamic fragility and kinetic fragility have the same order for the different liquids. By this definition, a liquid for which there is a more rapid drop in $\Delta S / \Delta S_{\mathrm{f}}$ with decreasing $T / T_{\mathrm{f}}$ is more thermodynamically fragile. Two thermodynamic fragility parameters were assumed [12]. One is $F_{1 / 2 \text {,cal }}=T_{1 / 2} / T_{\mathrm{m}}$, with $T_{1 / 2}$ being the temperature at which $\Delta S / \Delta S_{\mathrm{f}}=1 / 2$. The other is $F_{0.8}$, which is the fraction of fusion entropy lost $\left(1-\Delta S / \Delta S_{\mathrm{f}}\right)$ by $T / T_{\mathrm{m}}=0.8$ when cooling. Figure 4(a) shows the excess entropy $\Delta S$ normalized by $\Delta S_{\mathrm{f}}$ versus $T$ normalized by $T_{\mathrm{m}}$. It is seen that $F_{1 / 2 \text {,cal }}$ and $F_{0.8}$ coincide; thus, we only calculate $F_{0.8}$, as listed in Table 3 .

In Figure 4(a), however, we find that $F_{0.8}$ does not show the rapidity of the $\Delta S$ decrease thoroughly owing to the appearance of crossing points. $\Delta S$ for La-4, with $F_{0.8}(0.227)$ close to that for La-2 $(0.237)$ and that for La-5 (0.232), has a rate of decrease in the scaled excess entropy similar to that of La-1 (0.199) and that of La-3 (0.182). In Ref. [12], there is no crossing point between any two scaled excess entropy curves, and the larger $F_{0.8}$ results from a more rapid drop of $\Delta S / \Delta S_{\mathrm{f}}$ with diminishing $T / T_{\mathrm{m}}$ and thus larger $T_{K} / T_{\mathrm{m}}$. Generally, this is an ambiguous definition. Breakdowns of the coherence between this kind of thermodynamic fragility and kinetic fragility have been observed for molecular glassforming liquids [13].

Similar to Ref. [12], Martinez and Angell [14] plotted excess entropy $\Delta S$ scaled by $\Delta S_{\mathrm{g}}$ (excess entropy at $T_{\mathrm{g}}$ ) versus $T_{\mathrm{g}} / T$, which is similar to the Angell plot for kinetic fragility [1]. Following the idea of the mode of kinetic fragility $F_{1 / 2}=2 T_{\mathrm{g}} / T_{1 / 2}-1$ [30], a thermodynamic fragility parameter $F_{3 / 4}=2 T_{\mathrm{g}} / T_{3 / 4}-1$ is proposed, with $T_{3 / 4}$ the temperature at which $\Delta S_{\mathrm{g}} / \Delta S=3 / 4$ [14]. In Figure 4(b), we plot $\Delta S_{\mathrm{g}} / \Delta S$ versus $T_{\mathrm{g}} / T$ and mark the $\Delta S_{\mathrm{g}} / \Delta S=3 / 4$ line. To distinguish from $F_{0.8}$ defined in Ref. [12], we write the thermodynamic fragility parameter defined by $\Delta S_{\mathrm{g}}$ scaled entropy in Ref. [14] as $G_{3 / 4}$, and the values are listed in Table 3 .

Thermodynamic fragilities $F_{0.8}$ and $G_{3 / 4}$ and kinetic fragility $m$ are compared in Figure 5(a). Both $F_{0.8}$ and $G_{3 / 4}$ are positively correlated with the kinetic fragility for the most

Table 3 Thermodynamic and kinetic fragility indexes of La-based alloys

\begin{tabular}{llllll}
\hline Alloys & $m$ & $F_{0.8}$ & $G_{3 / 4}$ & $m_{\Delta S}$ \\
\hline La-1 & 33 & 0.199 & 0.647 & 0.742 \\
La-2 & 36 & 0.237 & 0.776 & 0.886 \\
La-3 & 42 & 0.182 & 0.715 & 0.09 \\
La-4 & 41 & 0.227 & 0.8 & 3.58 \\
La-5 & 38 & 0.232 & 0.68 & 0.975 & 2.25 \\
La-6 & 29 & 0.122 & 0.626 & 0.822 & 0.402 \\
\hline
\end{tabular}



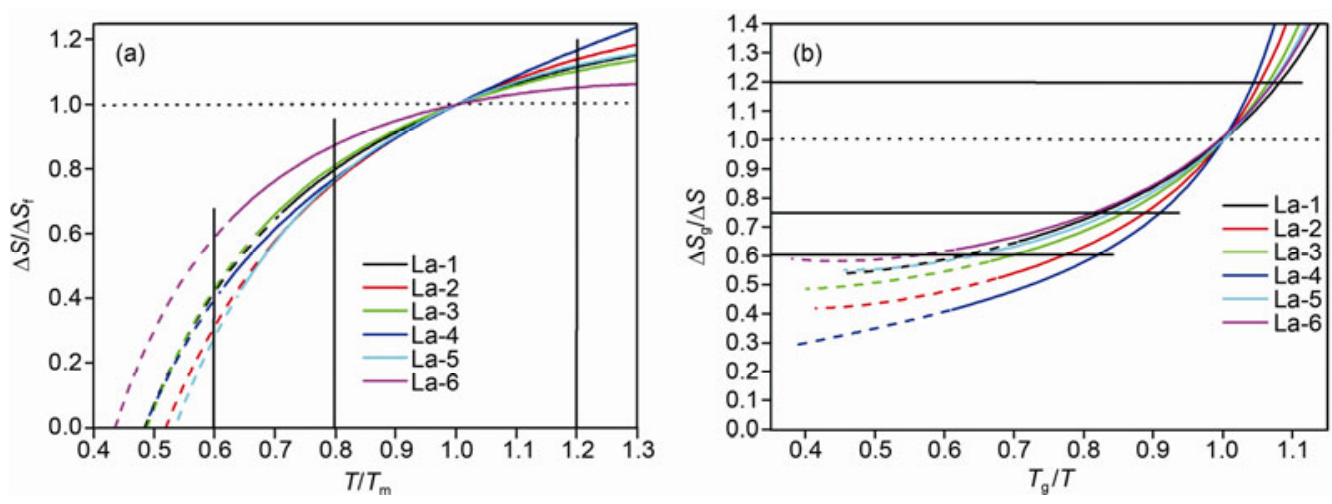

Figure 4 (a) Kauzmann plot of $\Delta S / \Delta S_{\mathrm{f}}$ against scaled temperature $T / T_{\mathrm{m}}$, with extrapolation below $T_{\mathrm{g}}$ to $T_{\mathrm{k}}$ (dashed lines) and above $T_{\mathrm{m}}$, in which the intercept lines at $T / T_{\mathrm{m}}=0.6,0.8$ and 1.2 are marked. (b) $S_{\mathrm{g}}$-scaled excess entropy versus $T_{\mathrm{g}}$-scaled temperature, with extrapolation above $T_{\mathrm{m}}$ (dashed lines) and below $T_{\mathrm{g}}$, in which the intercept lines at $\Delta S / \Delta S_{\mathrm{f}}=0.6,0.75$ and 1.2 are marked.

part, although the correlation with $G_{3 / 4}$ appears better. Since both lie central to the fragility range from $T_{\mathrm{g}}$ to $T_{\mathrm{m}}[14,30]$, the difference between the trends of $F_{0.8}$ and $G_{3 / 4}$ stems from the different values of the scaled excess entropy $\Delta S$, at $T_{\mathrm{g}}$ and $T_{\mathrm{m}}$. Both $T_{\mathrm{g}}$ and $T_{\mathrm{m}}$ vary for different metallic glasses, and variation in one characteristic temperature is thus ignored in the two definitions.

$\Delta S_{\mathrm{f}} /\left(T_{\mathrm{m}}-T_{\mathrm{k}}\right)$ [11], the average entropy-temperature dependence from $T_{\mathrm{m}}$ to $T_{\mathrm{k}}$, has been found to positively correlate with kinetic fragility in metallic glass-forming liquids. For the index of $\Delta S_{\mathrm{f}} /\left(T_{\mathrm{m}}-T_{\mathrm{k}}\right)$, if the variation in entropy for different glasses is eliminated by scaling the index with $\Delta S_{\mathrm{f}}$, as in the method employed to deduce thermodynamic fragilities in Refs. [12] and [14], then $1 /\left(T_{\mathrm{m}}-T_{\mathrm{k}}\right)$ or the reduced isentropic temperature $T_{\mathrm{k}} / T_{\mathrm{m}}$ seems to coincide with the original intention of thermodynamic fragility definition of the entropy slope $[11,12,14]$.

As in the definition of kinetic fragility $m$, we suppose that thermodynamic fragility $m_{\Delta S}$ can be represented by the drop in excess entropy approaching $T_{\mathrm{g}}$, which can be written as

$$
m_{\Delta S}=\left.\frac{\mathrm{d}\left(\Delta S_{\mathrm{g}} / \Delta S\right)}{\mathrm{d}\left(T_{\mathrm{g}} / T\right)}\right|_{T=T_{\mathrm{g}}} .
$$

Another concept of fragility, the fragility of superheated melts $M$ [2], is defined as the rate of change in viscosity at

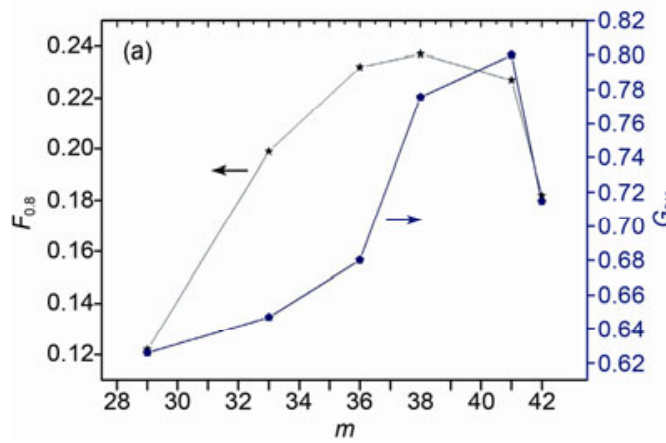

the liquidus temperature. $M$ is an index of the kinetic structure sensitivity to the temperature change approaching the phase transition of solidification [2], while $m$ corresponds to the glass phase transition. Fusion and solidification are opposing phase transitions. Thus, thermodynamic fragility $M_{\Delta S}$, reflecting the change rate of excess entropy approaching solidification/fusion, can be assumed according to Figure 4(a) as

$$
M_{\Delta S}=\left.\frac{\partial \Delta S / \Delta S_{\mathrm{f}}}{\partial T / T_{\mathrm{m}}}\right|_{T=T_{\mathrm{m}}}
$$

Values of $m_{\Delta S}$ and $M_{\Delta S}$ are listed in Table 3. We plot the relations between the two indexes and $m$ in Figure 5(b).

It is interesting that we find nearly linear proportional correlations between $M_{\Delta S}$ and $m$. The correlation between $M_{\Delta S}$ (i.e., the excess entropy slope at $T_{\mathrm{m}}$ instead of the excess entropy slope below $T_{\mathrm{m}}$ ) and $m$ may provide more detailed information about the correlation between the thermodynamics and kinetics of a liquid, which is discussed in the next section.

\section{Discussion}

Fragility was originally so named to infer that fragile liquids experience rapid breakdown of structure with decreasing

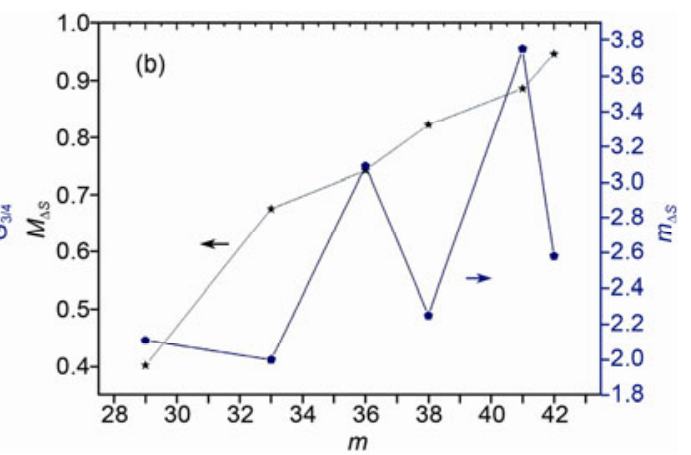

Figure 5 Correlation of the thermodynamic and kinetic fragilities: (a) $F_{0.8}$ and $G_{3 / 4}$ versus $m$, (b) $M_{\Delta S}$ and $m_{\Delta S}$ versus $m$. 
temperature approaching $T_{\mathrm{g}}$ [1]. In contrast to being "fragile", a liquid with slow structure change is deemed "strong". The Adam-Gibbs theory [9] predicts a dependence of a transition probability property on the configurational entropy $S_{\mathrm{c}}$ as

$$
\eta(T), \tau(T) \cong A \exp \left(C / T S_{\mathrm{c}}\right)
$$

where $S_{\mathrm{c}}$ is the configurational entropy, and $A$ and $C$ are constants without temperature dependence. The relation is based on the assumption of cooperatively rearranging regions. When configurational entropy $S_{\mathrm{c}}$ increases, the cooperative rearrangements of the structure can take place independently in smaller and smaller regions of the liquid. It has often been assumed that $S_{\mathrm{c}}$ can be approximated by the difference in entropy between liquid and crystal [12-14,23$27,30]$. Thus, the gradient of structural changes can be quantified in terms of $\Delta S^{\text {liq-cry }}$, and this is testified by the coincidence between $F_{0.8}$ and $G_{3 / 4}$ with $m$ in the La-based glass-formation liquid.

Besides $m$ (i.e., the slope of an Angell plot), the intercept of the Angell plot can be employed to quantify fragility $[12,23,31]$. The intercept is recommended as being a more reliable metric of the deviation from Arrhenius behavior, because it is central to the fragility range from $T_{\mathrm{m}}$ to $T_{\mathrm{g}}$. Following this idea, the intercept of scaled excess entropy was chosen to represent thermodynamic fragility [12,14] as $F_{0.8}$ and $G_{3 / 4}$, which we have calculated. However, the difference between the scaled $\Delta S$ plot and Angell plot, which may introduce randomness in the thermodynamic fragility, was neglected. In the Angell plot, the decreasing scaled viscosity or relaxation time curves split from an intersection point at $T_{\mathrm{g}}$, and join up again as the temperature approaches infinity (i.e., as the scaled temperature approaches zero). Figure 4(a) and (b) shows that the scaled excess entropy curves do not cross again. Thus, for an Angell plot, the variations in the intercept and slope coincide, while for the excess entropy plot, the correlation is to a certain extent random. We calculated the slope of scaled excess entropy at both ends of the fragility range and the average slope as thermodynamic fragility indexes. The thermodynamic fragility $M_{\Delta S}$ has high unexpected coherence with the kinetic fragility $m$, relative to that for $m_{\Delta S}$ or the average slope.

The excess entropy that we have used to explain the coincidence of $F_{0.8}$ and $G_{3 / 4}$ with $m$ is not the quantity that should appear in the Adam-Gibbs equation, even though it has been used in most experimental tests and the AdamGibbs equation usually tests well using the easily accessible excess entropy. The excess entropy should be divided into configurational entropy $S_{\mathrm{c}}$ and vibrational entropy $S_{\mathrm{vib}}$, as in Figure 6 [3]. The potential energy landscape (PEL) is an ideal concept for combining dynamics and thermodynamics, and is advantageously used to analyze and explain the phenomenology of the supercooled liquid state $[12,31,32]$. As shown in Figure 6, in the case of constant basin shape

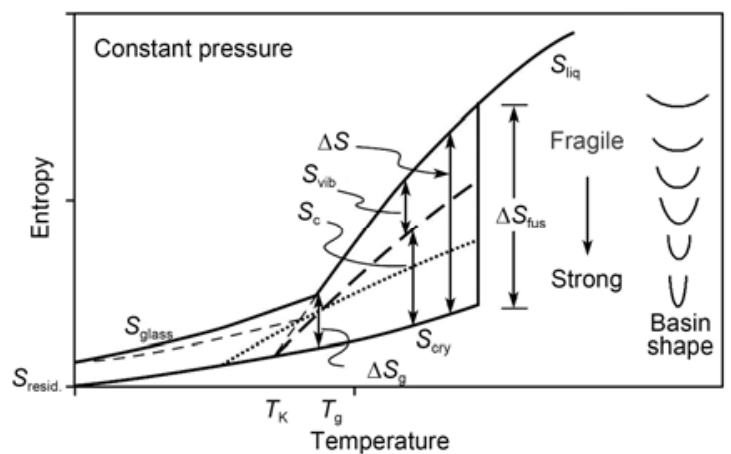

Figure 6 Changes in entropy of a system due to the change in the energy landscape ("basin shape") during cooling.

(strong liquid), the drive to higher enthalpy comes only from the configurational entropy; for variable basin shape (fragile liquid), the increased rate of excitation of vibrational entropy increases the rate of generation of $S_{\mathrm{c}}$ entropy, as well as that of the excess entropy [12].

By studying the PEL of glass-forming alloys, $\mathrm{Hu}$ et al. [33] found that the absolute value of chemical mixing enthalpy $\left|\Delta H_{\text {mix }}\right|$ is negatively related to the height of energy barriers separating the minima $\Delta \mu$. The height of energy barriers between minima on the PEL determines when a system freezes, and a larger $\Delta \mu$ indicates a more difficult change in the structure of the system. We calculate $\left|\Delta H_{\text {mix }}\right|$ on the basis of the extended regular solution model [34] and plot the $M_{\Delta S}-\left|\Delta H_{\text {mix }}\right|$ relationship in Figure 7. A positive correlation is found between $M_{\Delta S}$ and $\left|\Delta H_{\text {mix }}\right|$, and liquid with smaller $M_{\Delta S}$ thus has larger $\Delta \mu$. Therefore, the alloy with a slower change in thermodynamics at the start of the fragility range below $T_{\mathrm{m}}$ (a smaller $M_{\Delta S}$ ) has a more difficult change in structure approaching $T_{\mathrm{g}}$.

The coherence of thermodynamic fragility $M_{\Delta S}$ and kinetic fragility $m$ and $\Delta \mu$ suggests the possibility of a thermodynamic prediction of the structure stability. During rapid quenching, which generates BMGs, the drop in entropy at $T_{\mathrm{m}}$ (i.e., the beginning of the fragility range) plays a

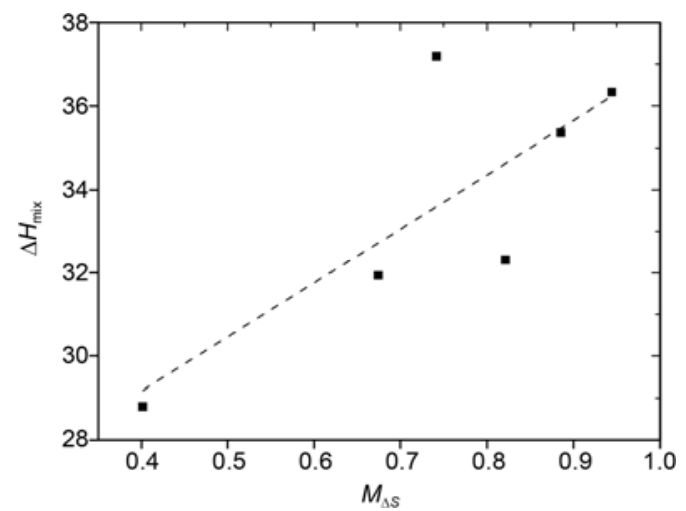

Figure 7 Calculated $\left|\Delta H_{\text {mix }}\right|$ versus $M_{\Delta S}$ for La-based BMGs (the dashed line is the linear fitting curve). 
crucial role in determining the structural stability of glassforming liquid in the fragility range.

\section{Conclusion}

Absolute values of the specific heat capacity $C_{\mathrm{p}}$ of six Labased BMGs were measured with respect to sapphire standards for the crystal, supercooled liquid, and melts. The differences in entropy $\left(\Delta S^{\text {liq-cry }}\right)$ and enthalpy $\left(\Delta H^{\text {liq-cry }}\right)$ between liquid and crystal as functions of temperature were calculated with $C_{\mathrm{p}}$ and the fusion heat $\Delta H_{\mathrm{f}}$. Thermodynamic fragility having different definitions was calculated and discussed for La-based glass-forming liquid on the basis of the $\Delta S^{\text {liq-cry }}$-temperature dependence. In La-based glassforming liquid, using the interception of scaled excess entropy $\Delta S$ curves as fragility indexes may result in randomness and ambiguity. We put forward thermodynamic fragilities $m_{\Delta S}$ and $M_{\Delta S}$ by evaluating the slope of the temperature dependence of the excess entropy at $T_{\mathrm{g}}$ and $T_{\mathrm{m}}$ respectively. The correlation between thermodynamic and kinetic fragilities was discussed on the basis of Adam-Gibbs and potential energy landscape theories. The thermodynamic fragility of the melt $M_{\Delta S}$, rather than that of the supercooled liquid $m_{\Delta S}$, relates linearly with kinetic fragility $m$ and the height of energy barriers separating the minima $\Delta \mu$.

This work was supported by the National Basic Research Program of China (2007CB613901), the National Natural Science Foundation of China (50871062 and 50831003) and the Natural Science Foundation of Shandong Province (Z2008F08).

1 Angell C A. Spectroscopy simulation and scattering, and the medium range order problem in glass. J Non-Cryst Solids, 1985, 73: 1-17

2 Bian X F, Sun B A, Hu L N, et al. Fragility of superheated melts and glass-forming ability in Al-based alloys. Phys Lett A, 2005, 335: 61-67

3 Song K K, Bian X F, Lv X Q, et al. The correlations among the fragility of supercooled liquids, the fragility of superheated melts, and the glass-forming ability for marginal metallic glasses. J Appl Phys, 2009, 105: 024304

4 Jia R, Bian X F, Lv X Q, et al. The relationship between viscosity and glass forming ability of Al-(Ni)-Yb alloy systems. Sci China Phys Mech Astron, 2010, 53: 390-393

5 Meng Q G, Zhou J K, Zheng H X, et al. Fragility of superheated melts and glass-forming ability in Pr-based alloys. Scripta Mater, 2006, 54: 777-781

6 Guo J, Bian X F, Li X L, et al. Correlation between superheated liquid fragility and potential energy landscape in Gd- and Pr-based glassforming alloys. Sci China Ser G-Phys Mech Astron, 2008, 51: 387-393

7 Guo J, Bian X F. A correlation between superheated liquid fragility and supercooled liquid fragility in La- and Sm-based glass-forming alloys. J Alloy Compd, 2010, 504: 205-207

8 Kauzmann W. The nature of the glassy state and the behavior of liquids at low temperatures. Chem Rev, 1948, 43: 219-256

9 Adam G, Gibbs J H. On the temperature dependence of cooperative relaxation properties in glass-forming liquids. J Chem Phys, 1965, 43: 139-146
10 Huang D H, McKenna G B. New insights into the fragility dilemma in liquids. J Chem Phys, 2001, 114: 5621-5630

11 Fan G J, Choo H, Liaw P K. Fragility of metallic glass-forming liquids: A simple thermodynamic connection. J Non-Cryst Solids, 2005, 351: 3879-3883

12 Ito K, Moynihan C T, Angell C A. Thermodynamic determination of fragility in liquids and a fragile-to-strong liquid transition in water. Nature, 1999, 398: 492-495

13 Ngai K L, Yamamuro O. Thermodynamic fragility and kinetic fragility in supercooling liquids: A missing link in molecular liquids. J Chem Phys, 1999, 111: 10403-10406

14 Martinez L M, Angell C A. A thermodynamic connection to the fragility of glass-forming liquids. Nature, 2001, 410: 663-667

$15 \mathrm{Ma} \mathrm{H}$, Fecht H J. Thermodynamic and kinetic fragilities of Mg-based bulk metallic glass-forming liquids. J Mater Res, 2008, 23: 2816-2820

16 Fan G J, Fecht H J. A cluster model for the viscous flow of glass-forming liquids. J Chem Phys, 2002, 116: 5002-5006

17 Tanaka H. Relation between thermodynamics and kinetics of glassforming liquids. Phys Rev Lett, 2003, 90: 055701

$18 \mathrm{Lu} \mathrm{Z} \mathrm{P,} \mathrm{Goh} \mathrm{T} \mathrm{T,} \mathrm{Li} \mathrm{Y.} \mathrm{Glass} \mathrm{formation} \mathrm{in} \mathrm{La-based} \mathrm{La-Al-Ni-}$ $\mathrm{Cu}-(\mathrm{Co})$ alloys by Bridgman solidification and their glass forming ability. Acta Mater, 1999, 47: 2215-2224

$19 \mathrm{Lu} \mathrm{Z} \mathrm{P,} \mathrm{Hu} \mathrm{X,} \mathrm{Li} \mathrm{Y,} \mathrm{et} \mathrm{al.} \mathrm{Glass} \mathrm{forming} \mathrm{ability} \mathrm{of} \mathrm{La-Al-Ni-Cu} \mathrm{and} \mathrm{Pd-}$ Si-Cu bulk metallic glasses. Mater Sci Eng A, 2001, 304-306: 679-682

20 Lu Z P, Hu X, Li Y. Thermodynamics of La based La-Al-Cu-Ni-Co alloys studied by temperature modulated DSC. Intermetallics, 2000, 8: $477-480$

21 Inoue A, Nakamure T, Sugita T, et al. Bulky La-Al-Tm (Tm=transition metal) amorphous alloys with high tensile strength produced by a high-pressure die casting method. Mater Trans JIM, 1993, 34: 351-358

22 Legg B A, Schroers J, Busch R. Thermodynamics, kinetics, and crystallization of $\mathrm{Pt}_{57.3} \mathrm{Cu}_{14.6} \mathrm{Ni}_{5.3} \mathrm{P}_{22.8}$ bulk metallic glass. Acta Mater, 2007, 55: 1109-1116

23 Kubaschewski O, Alcock C B, Spencer P J. Materials Thermochemistry. 6th ed. New York: Permagon, 1993

24 Glade S C, Busch R, Lee D S, et al. Thermodynamics of $\mathrm{Cu}_{47} \mathrm{Ti}_{34}$ $\mathrm{Zr}_{11} \mathrm{Ni}_{8}, \mathrm{Zr}_{52.5} \mathrm{Cu}_{17.9} \mathrm{Ni}_{14.6} \mathrm{Al}_{10} \mathrm{Ti}_{5}$ and $\mathrm{Zr}_{57} \mathrm{Cu}_{15.4} \mathrm{Ni}_{12.6} \mathrm{Al}_{10} \mathrm{Nb}_{5}$ bulk metallic glass forming alloys. J Appl Phys, 2000, 87: 7242-7248

25 Busch R, Liu W, Johnson W L. Thermodynamics and kinetics of the $\mathrm{Mg}_{65} \mathrm{Cu}_{25} \mathrm{Y}_{10}$ bulk metallic glass forming liquid. J Appl Phys, 1998, 83: 4134-4141

26 Wilde G, Gorler G P, Willnecker R, et al. Thermodynamic properties of $\mathrm{Pd}_{40} \mathrm{Ni}_{40} \mathrm{P}_{20}$ in the glassy, liquid, and crystalline states. Appl Phys Lett, 1994, 65: 397-399

27 Busch R, Kim Y J, Johnson W L. Thermodynamics and kinetics of the undercooled liquid and the glass transition of the $\mathrm{Zr}_{41.2} \mathrm{Ti}_{13.8} \mathrm{Cu}_{2.5^{-}}$ $\mathrm{Ni}_{10.0} \mathrm{Be}_{22.5}$ alloy. J Appl Phys, 1995, 77: 4039-4043

28 Crowley K J, Zografi G. The use of thermal methods for predicting glass-former fragility. Thermochim Acta, 2001, 380: 79-93

29 Busch R, Bakke E, Johnson W L. Viscosity of the supercooled liquid and relaxation at the glass transition of the $\mathrm{Zr}_{46.75} \mathrm{Ti}_{8.25} \mathrm{Cu}_{7.5} \mathrm{Ni}_{10} \mathrm{Be}_{27.5}$ bulk metallic glass forming alloy. Acta Mater, 1998, 46: 4725-4732

30 Richert R, Angell C A. Dynamics of glassforming liquids. IV: On the link between molecular dynamics and configurational entropy. J Chem Phys, 1998, 108: 9016-9026

31 Angell C A. Thermodynamics: Liquid landscape. Nature, 1998, 393: 521-524

32 Sastry S. The relationship between fragility, configurational entropy and the potential energy landscape of glass-forming liquids. Nature, 2001, 409: 164-167

33 Hu L N, Bian X F, Wang W M, et al. Thermodynamics and dynamics of metallic glass formers: Their correlation for the investigation on potential energy landscape. J Phys Chem B, 2005, 109: 13737-13742

34 Takeuchi A, Inoue A. Calculations of mixing enthalpy and mismatch entropy for ternary amorphous alloys. Mater Trans JIM, 2005, 41: 1372-1378

Open Access This article is distributed under the terms of the Creative Commons Attribution License which permits any use, distribution, and reproduction in any medium, provided the original author(s) and source are credited. 